\title{
A Soft Classification Model for Vendor Selection
}

\author{
Arpan K. Kar, Ashis K. Pani, Bijaya K. Mangaraj, and Supriya K. De
}

\begin{abstract}
This study proposes a pattern classification model for usage in the vendor selection problem. The usage of classification techniques has been an area of interest in decision sciences since ages. The major challenge in classification models is that they need huge amount of data points to train the models properly and hence are not suitable for adaptation in many business applications where the number of available data points to train similar decision support models is too less. A classification technique using fuzzy linear programming and fuzzy analytic hierarchy process has been proposed in this paper which addresses this challenge for providing decision support in the vendor selection problem where data availability is a challenge.
\end{abstract}

Index Terms-Vendor selection, supplier selection, classification, analytic hierarchy process, fuzzy linear programming.

\section{INTRODUCTION}

A pattern classification problem is essentially mapping an input pattern, represented as an input vector, to a particular class or category. Thus given a database $D=\left\{t_{1}, t_{2}, \ldots, t_{n}\right\}$ and a set of classes $C=\left\{C_{1}, \ldots, C_{m}\right\}$, the classification problem is to define a mapping $f: D \rightarrow C$ where each $t_{\mathrm{i}}$ is assigned to one class [9]. Traditionally classification has been studied using non-parametric techniques, Bayesian decision theory and parameter estimation, linear discriminant functions, stochastic methods, neural networks, and non-metric methods [1].

Recognizing pattern in data has been the area of study for a very long time, as early as 1960 s and pattern classification emerged as a major decision support technique in the early 1970s. Typically, pattern classification has been studied to map data into well-defined classes. A major problem with most classification techniques is that they require a very high amount of data to train the system, before it can be used to classify the rest of the data. This becomes a challenge in many business applications where it is very difficult to have that many data points, and yet would benefit if a classification system could be used to provide decision support for such a business setup.

One such area is the vendor selection problem in e-procurement which is an extremely important activity in integral supply chain management since purchasing is the primary point of contact with most of the supply-chain partners. While evaluating vendors who applied for a tender, typically decision support may be needed to select vendors from a group of as few as 30 to as many as few hundreds of

Manuscript received May 12, 2011; revised August 30, 2011.

Authors are with XLRI School of Business and Human Resources, Jamshedpur, Jharkhand, India (email: akpani@xlri.ac.in). vendors (approximate range) in the same company. Vendors are evaluated for selection on a number of dimensions for the same. Now, traditional techniques for decision support cannot handle a range of so few data points to few hundreds of data points. Also the nature of the data is both qualitative and quantitative and the responses for evaluation are taken over a wide variety of scales for the vendor selection criteria. Thus arises a need of a integrated approach of techniques to provide decision support for this problem.

This paper proposes a novel classification technique for usage in vendor selection and in similar multi-criteria decision making business problems such that these challenges of the problem domain may be better addressed.

\section{LITERATURE REVIEW}

\section{A. Classification with Fuzzy Linear Programming}

The recognition of pattern in data points across one or many dimensions was the first step in the developments in this domain. Pattern recognition started gaining prominence in the 1960s and slowly the importance of being able to predict data points based on their pattern of dimensions (characteristic) started gaining importance. These were the precursor to pattern classification based decision support models. Most classification based decision support models have been designed using non-parametric techniques, Bayesian decision theory and parameter estimation, linear discriminant functions, stochastic methods, neural networks, and non-metric methods, while more recently techniques using linear programming methods, fuzzy set theory and neural networks gained in prominence. Since classification is, in its very core, the task of recovering the model that generated the pattern, and then predict based on the recognition of that model, different classification techniques are useful depending on the type of candidate models themselves and the type of data available for the same [1].

The mathematical model for the pattern classification problem with linear programming was first conceptualized using maximized minimum distance and minimized sum of distance [2]. Later this was further improved with a hybrid model to take care of both the maximum sum of distance and minimized sum of distance in the same objective function, so as to get a compromised optimal solution [3]. Further built on this model, a multiple criteria linear programming method was proposed to get a compromised solution with conflicting goals that of minimizing minimized sum of distance and maximizing maximized minimum distance using goal programming [4]. Next, a fuzzy linear programming approach was developed for minimizing minimized sum of distance and maximizing maximized minimum distance to get an optimized but compromised solution [5]. Built on this 
work, a multiple criteria linear programming model with soft constraints and soft criteria was proposed for the purpose of classification of data points into soft sets [6]. The proposed model took care of the subjectivity of constraints and criteria while classifying data points into defined classes.

The current study extends upon the multiple criteria linear programming model with soft constraints and soft criteria [6] and extends it to classify vendors in an e-procurement scenario, into two soft sets, namely suitable and unsuitable classes. For defining the decision making vector, analytic hierarchy process has been integrated with the fuzzy linear programming model to ensure the relative importance of the criteria is taken into account in the decision making process. Finally, the decision support model helps to classify the vendors into two overlapping soft classes, namely, "suitable" and "unsuitable". For a particular firm, it will make business sense and create more value for the firm, if it gets its supplies from the class of "suitable" vendors. Similarly, it will not benefit that firm, if the same activities are carried out with "unsuitable" vendors.

\section{B. Analytic Hierarchy Process}

A novel approach has been taken in this paper for deciding the weights of the decision making vector, later denoted as $\mathrm{X}$ in this paper. For deciding the weights, a fuzzy extension of the theory of the analytic Hierarchy Process (AHP) [7], has been used in this paper.

The AHP is a general theory of measurement. It is used to derive ratio scales from both discrete and continuous paired comparisons in multilevel hierarchic structures. These comparisons may be taken from actual measurements or from a fundamental scale that reflects the relative strength of preferences and feelings. The AHP has a special treatment to identify departure from consistency among responses and also a measurement of this departure. It has found its widest applications in multi-criteria decision making, in planning and resource allocation, and in conflict resolution. The popularity of the AHP is for its robustness in converting ordinal scale to ratio scale, while checking for the consistency of the measurement.

The vendor selection problem was first approached using the classic theory of AHP with a crisp approach [8]. The application of AHP to the vendor selection problem domain has also been illustrated by multiple studies using both the classical crisp extension of the theory and the fuzzy extension of the theory. While few the initial approaches [9] adopted a theory based on the crisp scale to the vendor selection issue, more recent approaches ([10], [11]) introduced an AHP further developed using fuzzy set theory. A recent review of literature [12] on the vendor selection problem established that the AHP is one of the more popular approaches used to solve the problems in this domain.

In this study, a fuzzy extension of the AHP has been adapted for usage to find the relative importance of not only the criteria for the decision making for vendor selection, but also the relative importance of the individual sub-criteria or items used for the measurement of each criterion, and then the same has been modeled into the decision making vector.

\section{Problem Domain And Contribution}

It was established that the adoption of new e-procurement processes have well defined benefits for the business by lowering the time taken in each of the four stages of a procurement process, namely "Definition of buying requirements", "Identification and pre-qualification of vendors", "Definition of contract agreements" and subsequently the "Evaluation and rating of vendors" phases, in order [13]. It has been argued that when e-procurement solutions are adopted, qualitative and quantitative performance data gain in importance for the evaluation and selection viable vendors, and these criteria would differ significantly from traditional ones, and new important skills and capabilities would need to be added. The current study seeks to provide decision support for the second stage, namely, "Identification and pre-qualification of vendors" stage where both qualitative and quantitative data is required to be analyzed by the decision support system.

In the e-procurement scenario, generally, multiple vendors quote for a tender when the same is floated by a company. All these success criteria provide a clear indication that lowering the possible number of total vendors who applied for a tender to the number of possible vendors who would actually be more suitable in the vendor prequalification stage would play a key role in the success of a e-procurement implementation process. It was indicated that the studies in decision support have a huge scope in adding value to the existing body of literature and to the business community [14] in the vendor selection domain.

Multiple studies have been conducted in the area of optimizing the vendor selection process to provide decision support. These studies use various techniques to optimize multiple criteria in the vendor selection process and choose one vendor who would be most effective and suitable for the company who is seeking tenders from multiple vendors. However very few techniques process both qualitative and quantitative data. Also, in the current scenario, choosing only one vendor may not be prudent for a firm. In such a context, the current study provides a methodology to classify vendors into two classes, suitable and unsuitable and thus address this issue.

Most classification techniques have a huge data requirement during the training phase itself, unlike this technique, and so those techniques are not very suitable for usage in this problem domain, where number of vendors or data points will not be very high. Also, by following this methodology, the training phase and as well as the prediction phase may also be substantially improved as the subjective judgment may be reduced in the training data itself.

This decision support model proposed in this study also ensures that the company will get a larger pool of vendors choosing who would be beneficial for the company, while negating tenders from vendors who would not be suitable for the company. Other studies in this problem domain developed techniques to identify the most suitable vendor. This again is not very beneficial for the firm owing to the increased bargaining power of that vendor. The proposed technique bridges this gap and fulfills the requirement of identifying multiple suitable vendors from a pool of vendors. Also the proposed decision support model based on pattern 
classification is different from most other prior decision support models in that it can analyze and select vendors using both qualitative and quantitative data dimensions.

Thus the current study aims in providing decision support to the vendor selection problem in the vendor prequalification stage, and thus lower critical response time in one of the four critical phases in e-procurement, as well as improve the decision making process.

\section{TECHNIQUe PROPOSAL}

For testing this proposed model, the initial criteria were chosen from existing literature ([6] and [15]) for usage; following a Delphi study to test the contextual relevance of the finally chosen criteria for vendor evaluation. The details of the same are explained in the research design section later in this paper. For the purpose of generalization of the proposed decision support model, let us assume that there are ' $r$ ' such attributes or criteria.

Let $\mathrm{A}_{\mathrm{ij}}$ be the data set consisting of the data regarding the $i$-th vendor and the $j$-th attribute of that particular vendor for $j$ ranging from 1 to $r$. Data about the $i$-th vendor will be denoted by $A_{i}$ which will essentially be a vector consisting of $r$ attributes. All the data would be numeric in nature, fuzzified to a value ranging from 0 to 1 . This would be achieved using the following equation:

$$
A_{i j}=\left(S_{s}-S_{\min }\right) / S_{\max }-S_{\min }
$$

Here $\mathrm{S}_{\mathrm{s}}$ is the response for the item on the scale, $\mathrm{S}_{\min }$ is the minimum acceptable response level giving the lowest satisfaction, and $S_{\max }$ is the minimum response level giving the highest satisfaction.

For this model, let $X$ be the decision making vector consisting of $\left(x_{1}, x_{2}, x_{3}, \ldots, x_{\mathrm{r}}\right)$ such that it provides decision support by providing the best coefficients for the attributes of the $\mathrm{i}$-th vendor. This is the decision making vector and it would be decided as described later, using the analytic hierarchy process.

First, using the fuzzy extension of the classic AHP theory [1], the relative importance of each criteria, i.e., the weights $W_{0}(i)$, for $i=1$ to 7 , and those of the sub-criteria, i.e., those of $W_{1}(i, j)$ for $i=1$ to 7 and $j=1$ to $n_{i}$, would be found out using the mentioned technique. Here there are $r$ main criteria, each with $\mathrm{n}_{\mathrm{i}}$ sub-criteria. The vector $X$ would consist of $\left(x_{1}, x_{2}\right.$, $\left.x_{3}, \ldots, x_{r}\right)$ such that $x_{i}$ would represent the product of $W_{0}(i, j)$ and $W_{1}(\mathrm{i})$, in order of sequence. Thus, essentially, $\mathrm{x}_{\mathrm{i}}$ would represent the relative importance of all the items in the scale chosen for vendor selection, such that $\sum x_{i}=1$.

TABLE I: AHP RESPONSE MAPPING SCALE

\begin{tabular}{|c|c|}
\hline Linguistic response type & Response value \\
\hline Equal importance & $\sim 1=(1,1,3)$ \\
\hline Moderate importance & $\sim 3=(1,3,5)$ \\
\hline Strong importance & $\sim 5=(3,5,7)$ \\
\hline Very strong importance & $\sim 7=(5,7,9)$ \\
\hline Extreme importance & $\sim 9=(7,9,9)$ \\
\hline
\end{tabular}

A single response is taken from the decision maker (e.g. a procurement expert in this case) by asking the decision maker to evaluate the relative importance of all the criteria for vendor evaluation, by making binary comparisons between two criteria at a time. The responses of the linguistic comparisons are mapped into fuzzy sets using the scale as described in Table I.

In this scale for receiving responses, a triangular function to fuzzily the linguistic responses has been used instead of the usual Saaty's crisp response. The triangular fuzzy function has been proposed for usage in this context as it has been assumed that there is equal probability of the response of the next level as there is to the response of the previous level, when a slightly vague linguistic comparison is made by a respondent. Therefore a fuzzy response of $[n]$ on the previously described fuzzy scale will have a value of $[(n-2$, $0.25),(n, 0.50),(n+2,0.25)]$, where $(x, \mu)$ is such that ' $x$ ' is the central element of the fuzzy set and ' $\mu$ ' is the degree of belongingness of the response to the element.

After taking the responses it would be coded by the AHP theory as defined by Saaty ${ }^{[8]}$ to check for consistency. Finally the fuzzy set of weights for the criteria would be modeled as per the rules built upon the model developed by Pani and $\operatorname{Kar}^{[1]}$ (2011). This would provide an accurate estimation of the relative weights of the criteria for the decision maker using fuzzy values. These fuzzy weights will be re-converted to crisp values using a weighted mean approach for the determination of the decision making vector $X$.

Now for this model, let us assume a degree of overlap among the two classes (sets), i.e. the boundaries are essentially soft. Now let $b$ be the boundary value for the two soft classes of suitable and unsuitable vendors, based on their scores in ' $r$ ' criteria. Essentially this value of $b$ is dependent on the value of the decision making vector $X$. Now let $\alpha_{i}$ be the overlapping of two-class boundary for vendor $A_{i}$ (external measurement) and $\alpha$ be the maximum overlapping of two-class boundary for all cases $A_{i}$ so that $\alpha_{i}<\alpha$ for all $\alpha_{i}$. Let $\beta_{i}$ be the distance of case $A_{i}$ from its adjusted boundary (internal measurement) and $\beta$ be the minimum distance for all cases Ai from its adjusted boundary so that $\beta_{i}>\beta$ for all $\beta_{i}$. For an ideal hard separation between the two classes, $\beta=0$ and let us assume this to be the minimum value of $\beta$.

Let $y_{1 L}$ be Minimized Sum of Distances (MSD) and $\mathrm{y}_{2 \mathrm{U}}$ be Maximized Minimum Distance (MMD), then one can assume that the value of Maximize $\Sigma_{i} \alpha_{i}$ to be $y_{1 U}$ and that of Minimize $\Sigma_{i} \beta_{i}$ to be $y_{2 L}$. Normally, the "upper bound" $y_{1 U}$ and the "lower bound" $y_{2 L}$ would not exist for the formulations but can be assumed to be a very large numeric value and 0 respectively for both the cases for all practical purposes, subject to $b$ varies in the range 0 to 1 .

Now we define two functions $F_{1}$ and $F_{2}$ to take care of the MSD and MMD criteria.

$$
\begin{aligned}
& \text { Let } F_{1}=\left\{x: y_{1 L} \leq \Sigma_{i} \alpha_{\mathrm{i}} \leq \mathrm{y}_{1 U}\right\} . \\
& \text { Let } F_{2}=\left\{x: y_{2 L} \leq \Sigma_{i} \beta_{i} \leq \mathrm{y}_{2 U}\right\}
\end{aligned}
$$

Let their membership functions can be expressed by as given by the equations in the following part:

$F_{1}$ is defined as follows:

$$
\begin{aligned}
& \mu F_{1}(x): 1 \quad \text { if } \Sigma_{i} \alpha_{\mathrm{i}} \geq y_{1 U} \\
& \mu F_{1}(x):\left(\Sigma_{i} \alpha_{i}-y_{1 L}\right) /\left(y_{1 U}-y_{1 L}\right) \quad \text { if } y_{1 L}<\Sigma_{i} \alpha_{i}<y_{1 U}
\end{aligned}
$$




$$
\mu F_{1}(x): 0 \quad \text { if } \Sigma_{i} \alpha_{i} \leq \mathrm{y}_{1 L}
$$

and $\mathrm{F}_{2}$ is defined as follows:

$$
\begin{aligned}
& \mu F_{2}(x): 1 \quad \text { if } \Sigma_{i} \beta_{i} \geq \mathrm{y}_{2 U} \\
& \mu F_{2}(x):\left(\Sigma_{i} \beta_{i}-\mathrm{y}_{2 L}\right) /\left(\mathrm{y}_{2 U}-\mathrm{y}_{2 L}\right) \quad \text { if } \mathrm{y}_{2 L}<\Sigma_{i} \beta_{i}<\mathrm{y}_{2 U} \\
& \mu F_{2}(x): 0 \quad \text { if } \Sigma_{i} \beta_{i} \leq \mathrm{y}_{2 L}
\end{aligned}
$$

Then the fuzzy set of the objective functions is $F=F_{1} \cap F_{2}$ and its membership function is $\mu F(x)=\min \left\{\mu F_{1}(x), \mu F_{2}(x)\right\}$. Using the crisp constraint set $X=\left\{x: A_{i} X=b+\alpha_{i}-\beta_{i}, A_{\mathrm{i}} \in\right.$ suitable; $A_{i} X=b-\alpha_{i}+\beta_{i}, \mathrm{~A}_{\mathrm{i}} \in$ unsuitable $\}$, the fuzzy set of the decision problem is $D=F_{1} \cap F_{2} \cap \mathrm{X}$, and its membership function is $\mu D(x)=\mu F_{1} \cap F_{2} \cap X(x)$.

Zimmermann has shown that the "optimal solution" of maximize $\mu D(x)=\max \min \left\{\mu F_{1}(x), \mu F_{2}(x), \mu X(x)\right\}$ is an efficient solution of model, and this problem is equivalent to the following linear program, which can be used for classification:

Maximize $\xi$

Subject to:

$$
\begin{aligned}
& \xi \leq\left(\sum_{i} \alpha_{i}-\mathrm{y}_{1 L}\right) /\left(y_{1 U}-y_{1 L}\right) \\
& \xi \leq\left(\Sigma_{i} \beta_{i}-\mathrm{y}_{2 L}\right) /\left(y_{2 U}-y_{2 L}\right) \\
& A_{i} X=b+\alpha_{i}-\beta_{i}, A_{i} \in \text { suitable, } \\
& A_{i} X=b-\alpha_{i}+\beta_{i}, A_{i} \in \text { unsuitable, }
\end{aligned}
$$

where $A i, y_{1} L, y_{1} U, y_{2} L$ and $y_{2} U$ are known, $X$ and $\mathrm{b}$ are unrestricted, and $\alpha \mathrm{i}, \beta_{i}, \xi \geq 0$.

We define this linear programming model as $M_{1}$.

Also we define a metric to calculate the actual classification error level e as follows:

$\mathrm{e}=[$ (wrongly classified as unsuitable but actually suitable vendor count $)+$ (wrongly classified as suitable but actually unsuitable vendor count)] / (total number of data points)

Now we utilize the following algorithm for the classification of the vendors into two classes, suitable and unsuitable.

1) First the data set is to be cleaned and checked for validity of the responses across the ' $r$ ' criteria for all the data points $A_{i}$, for ' $n$ ' vendors. Here, each vendor is a data point $\mathrm{A}_{\mathrm{i}}$, treated as a vector with ' $r$ ' dimensions.

2) Now the entire data set is divided into two data subsets, one to the train the method of size $n_{1}$, and the other would to test the data of size $\mathrm{n}_{2}$. The subsets are created such that $n_{1}+n_{2}=n$. Care should be taken so that the representation of both the suitable and unsuitable vendors in $\mathrm{n}_{1}$ is sufficient to obtain satisfactory results. A larger set of data during the training stage would improve the prediction capability of the decision support system.

3) An accepted error of classification (prediction), e-acc, is to be defined. The model would be said to be functioning properly if the prediction error is more than this accepted error of classification e-acc. Otherwise, the model would require more data points during the training stage.

4) Now we define the $X$ vector, using the values as has been defined earlier for each of the $x_{i}$ for $i=1$ to $r$, using the rules of the fuzzy AHP as described earlier.

5) Then we find out the maximum and the minimum of the sum of $\alpha_{i}$ and $\beta_{i}$, using only $\alpha_{i}$ and $\beta_{i}$ respectively in the constraints, against $A_{i} X$ while restricting the value of $\mathrm{b}$ from 0 to 1 . This will give two separate $b$-value for the two models based on the individual MSD and MMD models.

6) Now we use the training data set to compute $A_{i} X$ for the data and the values of maximum and the minimum of the sum of $\alpha_{i}$ and $\beta_{i}$, to compute the optimal value of " $b$ " for all the data points in $\mathrm{n}_{1}$.

7) The corresponding $b$ value is used with the $X$ vector to classify the second set of $n_{2}$ records.

8) If for the found out $b$ value, the classification error e exceeds e-acc, check the training data set to see whether sufficient representation is available from both the classes or add datasets to do so or else lower the e-acc value and restart the process from step 1 else stop.

In the first step, the data mart is prepared by cleaning the available data. In the second step, the data is divided into two subsets, such that, one may be used for training, while the other may be used for testing the capabilities of the methodology. The type of data in the training set would also affect the $b$ value, and hence the classification output in the next stage. Thus it would be necessary to have sufficient representation of both the classes in the training data set to obtain an ideal b-value, so that prediction of classes may be done optimally.

After the $b$ value is obtained, during classification phase, if the $b$ value is increased on the test data, it will serve to choose the better suitable vendors amongst all the suitable vendors. Thus, by changing the b-value, it will be possible to actually get the subset of the best " $n$ " vendors, from a class of suitable vendors. Conversely, if sufficient suitable vendors are absent, lowering the b-value nominally will present the decision maker with the set of the most suitable vendors amongst the otherwise unsuitable set of vendors.

\section{RESEARCH DESIGN}

For selecting the criteria which would be most relevant for the evaluation of vendors, a Delphi study [16] consisting of 30 respondents and 2 iterations was carried out. From the outcomes of the study, 7 criteria were finally chosen as being relevant for vendor evaluation in India, which have been described in the subsequent part. The seven criteria finally chosen were product quality, compliance with the delivery schedule, price quoted as compared to market average price, vendor's technological capability, vendor's production capability, vendor's financial position and finally the vendor's electronic transaction capability.

Based on these criteria, data was simulated for 50 respondent vendors following an exploratory study to identify possible vendor scores in each of the criteria, which were chosen earlier through the Delphi study. The data simulated was validated by a procurement manager from an iron and steel manufacturing company in India, to evaluate its representativeness of the suppliers of their firm, for the experimental setup.

The proposed classification model was programmed in Microsoft Excel 2007 using the solver add-on. The fuzzy AHP model was also programmed in Microsoft Excel 2007. Based on the previously simulated data, the procurement expert was asked to respond to a questionnaire for the fuzzy 
AHP so as to estimate his preferences of the relative importance of the seven supplier evaluation criteria.

The same expert was then asked to identify suitable and unsuitable vendors from that pool of 50 vendors, based on the simulated responses for the mentioned seven criteria identified from the Delphi study. The first 20 data points were used to train the proposed model and the next 30 data points were used to check the validity of the model in predicting the suitability of vendors.

\section{RESUlTS}

After training the model, the maximum and the minimum value of the summation of $\alpha_{i}$ came to be 10.88 and 2.34 respectively. Similarly the maximum and the minimum value of the summation of $\beta_{i}$ came to be 5.28 as the maximum and 0 was assumed as the minimum possible degree of overlap of boundaries, i.e. the case of no overlap.

After training the model, for the given simulated dataset, the optimal $b$-value from the training set came at $b=0.58$. Using that $b$-value, the rest of the data was analyzed and the vendor acceptance was predicted for 30 more data points. Out of the 30 data points, only 4 data points failed to be classified according to the decision maker's class. Thus the classification error was $13 \%$.

Considering only 20 data points were used to train the data in the first phase, the prediction capability is sufficiently dependable for a practical application in the problem domain. Also, it is important to note that those which had been wrongly classified by the model from the class as decided the decision maker; only marginally suitable vendors had been rejected by the model as being suitable. Therefore from the point of utility for a business level application, the model actually would reject those vendors who would not be very good as per the criteria and their relative weights, as decided by the decision maker, i.e. the procurement management in this case. Thus, only those vendors would actually be chosen by the model who would be relatively very suitable for the particular requirement in the company. However, it is important to note that the success of such a decision support model would depend a lot on the type of data that would be available in the training phase.

\section{DISCUSSION}

In this paper, a heuristic classification scheme using fuzzy linear programming techniques and fuzzy AHP has been proposed for vendor classification in an e-procurement scenario. This approach is based on the time tested linear discriminant analysis using linear programming and multiple criteria linear programming classification. This proposed decision support model has the advantage that very few data points will be needed for training the system, unlike other classifiers where data requirement is very large. Also, it has the capability to use both quantitative data and qualitative data for the purpose of pattern recognition and subsequently pattern classification. Also it does not suffer from the limitation to provide decision support for a limited number of data points (suppliers).

By choosing the most suitable vendors through decision support through this model, the firm can ensure that the time spent on the prequalification of vendors will be minimized to a large extent by automating most of the processes. Also the selection process will be improved to a great extent through the adoption of such a process. The sole focus of this paper is to propose a decision support model for the procurement division of a company for choosing its vendors when multiple vendors float tenders for a particular call for tenders and no attempt has been made in this paper to redefine or add criteria for the evaluation of vendors. The study adapts already developed criteria and provides decision support to select suitable vendors from a pool of vendors for usage for the Indian manufacturing industries. Also, since this technique has been tested via an experiment with simulated data, a future research direction could be to test it on data from an actual business setting. Another future research direction could be to incorporate group decision support into the proposed classification model.

\section{REFERENCES}

[1] R. L. Nydick and R. P. Hill, "Using the analytic hierarchy process to structure the vendor selection procedure", International Journal of Purchasing Material Management, vol. 28, pp. 31-36, 1992.

[2] M. H. Dunham, "Data mining: Introductory and advanced topics", Pearson Education, 2003.

[3] E. Bottani, and A. Rizzi, "A fuzzy multi-attribute framework for vendor selection in an e-procurement environment", International Journal of Logistics: Research and Applications, vol. 8:3, pp. 249-266, 2005.

[4] Y. Shi, M. Wise, M. Luo, and Y. Lin, "Data Mining in Credit Card Portfolio Management: a Multiple Criteria Decision Making Approach", in Proc. Multiple Criteria Decision Making in the New Millennium, Berlin, Springer. 2001.

[5] F. Glover, "Improve Linear Programming Models for Discriminant Analysis", Decision Sciences, vol. 21, pp. 771-785, 1990.

[6] A. K. Pani, and A. K. Kar, "A Study to compare Relative Importance of Criteria for Vendor Evaluation in e-Procurement", in Proc. 44th Hawaii International Conference on System Sciences, Kauai, USA, 2011.

[7] T. L. Saaty, "The Analytic Hierarchy Process", New York, McGraw Hill International, 1980.

[8] P. Morlacchi, "Vendor evaluation and selection: the design process and a fuzzy hierarchical model”, in Proc. 8th IPSERA Conference, 1999.

[9] C. Kahraman, U. Cebeci, and Z. Ulukan, "Multi-criteria vendor selection using fuzzy AHP", Logistics Information Management, vol. 16, pp. 382-394, 2003

[10] N. Freed, and F. Glover, "Simple but Powerful Goal Programming Models for Discriminant Problems", European Journal of Operational Research, vol. 7:1, pp. 44-60, 1981.

[11] A. Petroni, and M. Braglia, "A quality-assurance oriented methodology for handling trade-offs in vendor selection", International Journal of Physical Distribution and Logistics Management, vol. 30, pp. 96-111, 2000.

[12] W. Ho, X. Xu, P. K. Dey, Multi-criteria decision making approaches for vendor evaluation and selection: A literature review, European Journal of Operational Research. Vol. 202, pp. 16-24, 2010.

[13] W. D. Presutti, "Supply management and e-procurement: creating value added in the supply chain", Industrial Marketing Management, vol. 32, pp. 219-226, 2003.

[14] T. Schoenherr, and V. M. R. Tummala, "Electronic procurement: a structured literature review and directions for future research", International Journal of Procurement Management, vol. 1: 1/2, pp. 8-37, 2007.

[15] C. Masella, and A. Rangone, "A contingent approach to the design of vendor selection systems for different types of co-operative customer/vendor relationships", International Journal of Operation \& Production Management, vol. 20, pp. 70-84, 2000.

[16] A. Li, Y. Shi, and J. He, "A Data Classification method based on Fuzzy Linear Programming”, in Proc. Multi-criteria Decision Making, Chania, Greece. 2006.

[17] E. Bottani, and A. Rizzi, "A fuzzy multi-attribute framework for vendor selection in an e-procurement environment", International Journal of Logistics: Research and Applications, vol. 8:3, pp. 249-266, 2005.

[18] J. He, X. Liu, Y. Shi, W. Xu, and N. Yan, "Classifications of Credit Cardholder Behavior by Using Fuzzy Linear Programming", Information Technology and Decision Making, Vol. 3, pp. 633-650, 2004. 